\title{
ON THE FEFFERMAN-PHONG INEQUALITY AND RELATED PROBLEMS
}

\author{
DANIEL TATARU
}

\begin{abstract}
The aim of this article is to provide a new approach for the classical Fefferman-Phong inequality and some other related inequalities concerning pseudodifferential operators. The idea is to conjugate these estimates with respect to the FBI transform and obtain equivalent formulations which require only a few derivatives of the symbol. Modulo some integration by parts, these are reduced to certain decomposition results for $C^{1,1}$, respectively $C^{3,1}$ functions. As a byproduct of this method we get to extend the inequalities to (possibly sharp) classes of symbols with limited smoothness.
\end{abstract}

\section{INTRODUCTION}

We say that a pseudodifferential operator $A$ is semipositive if it satisfies an inequality of the form

$$
\Re<A u, u>\geq-c\|u\|_{L^{2}}^{2}
$$

for all functions $u \in \mathcal{S}\left(\mathbb{R}^{n}\right)$. We consider pseudodifferential operators whose symbols are either scalar or $N \times N$ matrices. The classical formulation of the sharp Garding's inequality and of Fefferman-Phong's inequality is

Theorem 1. a) (sharp Garding [6][9]) Let $a(x, \xi) \in S^{1}$ be an $N \times N$ symbol satisfying $\Re a \geq 0$. Then $A(x, D)$ is semipositive.

b) (Fefferman-Phong [3]) Let $a(x, \xi) \in S^{2}$ be a real scalar nonnegative symbol. Then $A(x, D)$ is semipositive.

These results have been extended later on to various other classes of symbols (see [8] and references therein). However, here we aim to keep things uncomplicated so we restrict ourselves to the simplest symbol classes. The first result we prove is stated using the Weyl calculus:

Theorem 2. a) Let $a(x, \xi)$ be a real $N \times N$ nonnegative symbol in $T^{*} \mathbb{R}^{n}$ satisfying

$$
\left|\partial_{x}^{\alpha} \partial_{\xi}^{\beta} a(x, \xi)\right| \leq c_{\alpha \beta} \mu^{\frac{|\alpha|-|\beta|}{2}}, \quad|\alpha|+|\beta| \geq 2
$$

for some $\mu>0$. Then $A^{w}\left(\frac{x+y}{2}, D\right)$ is semipositive.

b) Let $a(x, \xi)$ be a real scalar nonnegative symbol in $T^{*} \mathbb{R}^{n}$ satisfying

$$
\left|\partial_{x}^{\alpha} \partial_{\xi}^{\beta} a(x, \xi)\right| \leq c_{\alpha \beta} \mu^{\frac{|\alpha|-|\beta|}{2}}, \quad|\alpha|+|\beta| \geq 4
$$

for some $\mu>0$. Then $A^{w}\left(\frac{x+y}{2}, D\right)$ is semipositive.

Research partially supported by the NSF grant DMS-9970297. 
For scalar symbols (b) is stronger than part (a). However from a historical standpoint it is useful to state them separately, as (a) corresponds to the sharp Garding's inequality and (b) corresponds to the Fefferman-Phong inequality. Note that we impose no restriction on the size of the support of the symbol $a$. Hence this is a scale invariant result with respect to the parameter $\mu$. This result is not new (see [1]), however we hope that the proof we present here is simpler and sheds some new light on this class of problems.

The classical proof [3] of the Fefferman-Phong inequality, refined in [1], is based on successive localizations combined on an inductive argument with respect to the dimension. All this is done at the operator level. The idea is to successively peel off squares of operators in a sufficiently localized setting, all while retaining sufficient orthogonality to be able to assemble together the localized results. Our idea, instead, is to reduce the problem to a statement about decompositions of $\dot{C}^{3,1}$ nonnegative functions as sums of squares of $\dot{C}^{1,1}$ functions:

Proposition 1.1. There exist $K, M>0$ depending only on the dimension $n$ so that for any nonnegative function $\phi$ in $\mathbb{R}^{n}$ satisfying

$$
\left|\nabla^{4} \phi\right| \leq 1
$$

there exist functions $\left\{\phi_{k}\right\}_{k=1 \ldots K}$ so that

$$
\phi=\sum_{k=1}^{K} \phi_{k}^{2}
$$

and

$$
\sum_{k}\left|\nabla^{2} \phi_{k}\right|^{2}+\left|\left(\nabla^{3} \phi_{k}\right)\left(\nabla \phi_{k}\right)\right| \leq M
$$

Note that the expression $\left(\nabla^{3} \phi_{k}\right)\left(\nabla \phi_{k}\right)$ above is well defined as a distribution by

$$
\left(\nabla^{3} \phi_{k}\right)\left(\nabla \phi_{k}\right)=\nabla\left(\left(\nabla^{2} \phi_{k}\right)\left(\nabla \phi_{k}\right)\right)-\left(\nabla^{2} \phi_{k}\right)\left(\nabla^{2} \phi_{k}\right) \text {. }
$$

The proof of this result mirrors to a certain extent the classical proof, but is considerably simpler and quite elementary.

By a standard Littlewood-Paley decomposition Theorem 2(a),(b) implies corresponding result for symbols in $S_{\rho, \rho}$ like classes, with $0 \leq \rho<1$. We introduce two pairs of conditions,

$$
\begin{gathered}
\left|\partial_{x}^{\alpha} \partial_{\xi}^{\beta} a(x, \xi)\right| \leq c_{\alpha \beta}(1+|\xi|)^{\rho(|\alpha|-|\beta|)}, \quad|\alpha|+|\beta| \geq 2, \\
\left|\partial_{x}^{\alpha} \partial_{\xi}^{\beta} a(x, \xi)\right| \leq c_{\alpha \beta}(1+|\xi|)^{2(1-\rho)+(2 \rho-1)|\alpha|-|\beta|}, \quad|\alpha|+|\beta|<2 .
\end{gathered}
$$

respectively

$$
\begin{gathered}
\left|\partial_{x}^{\alpha} \partial_{\xi}^{\beta} a(x, \xi)\right| \leq c_{\alpha \beta}(1+|\xi|)^{\rho(|\alpha|-|\beta|)}, \quad|\alpha|+|\beta| \geq 4, \\
\left|\partial_{x}^{\alpha} \partial_{\xi}^{\beta} a(x, \xi)\right| \leq c(1+|\xi|)^{4(1-\rho)+(2 \rho-1)|\alpha|-|\beta|}, \quad|\alpha|+|\beta|<4 .
\end{gathered}
$$


The important conditions here are (5) and (7). The only purpose of (6) and (8) is to insure that the dyadic pieces of $a$ still satisfy (5), respectively (7). Likely with some extra work one can elliminate (6) and (8) at least partially, if not fully (see also [1]).

Corollary 1.2. a) Let $a(x, \xi)$ be a real $N \times N$ nonnegative symbol which satisfies (5) and (6). Then $A^{w}\left(\frac{x+y}{2}, D\right)$ is semipositive.

b) Let $a(x, \xi)$ be a real scalar nonnegative symbol which satisfies (7) and (8). Then $A^{w}\left(\frac{x+y}{2}, D\right)$ is semipositive.

Part (a) easily implies a generalization of the sharp Garding's inequality,

Corollary 1.3. Let $a \in C^{2} S^{1}$ be an $N \times N$ symbol so that $\Re a \geq 0$. Then $A(x, D)$ is semipositive.

Part (b) instead gives a generalization of the Fefferman-Phong inequality,

Corollary 1.4. Let $a(x, \xi) \in C^{4} S^{2}$ be a real scalar nonnegative symbol. Then $A(x, D)$ is semipositive.

One can also get intermediate results by trading regularity for added decay of the symbol.

Corollary 1.5. Consider an $N \times N$ nonnegative symbol $a(x, \xi) \in C^{s} S^{\frac{2 s}{2+s}}$ for some $0 \leq s \leq 2$. Then $A(x, D)$ is semipositive.

Corollary 1.6. Consider a real scalar nonnegative symbol $a(x, \xi) \in C^{s} S^{\frac{4 s}{4+s}}$ for some $0 \leq s \leq 4$. Then $A(x, D)$ is semipositive.

The second problem we consider deals with the following question: when is a pseudodifferential operator "controlled" by some other pseudodifferential operators ? More precisely, consider real scalar symbols $b$ and $a_{j}$ so that $|b| \leq \sum\left|a_{j}\right|$. Then we can ask whether a similar bound holds for operators,

$$
\|B u\|_{L^{2}} \lesssim \sum\left\|A_{j} u\right\|_{L^{2}}+\|u\|_{L^{2}} .
$$

It is important here that there are several $A_{j}$ 's. If there is only one then the problem as stated is considerably simpler, and one can get better results, see [4]. In the case of smooth symbols $a_{j}, b \in S^{1}$ one can easily prove this using the Fefferman-Phong inequality. Using instead Corollary 1.4 one can show that a similar result holds for $C^{4} S^{1}$ symbols. However, in order to conjugate this result with respect to the FBI transform one only needs two derivatives on the symbol; this would seem to indicate that perhaps $C^{2} S^{1}$ should suffice. Indeed, we are able to prove the following scale invariant result:

Theorem 3. Let $\mu>0$ and $a_{j}$, b be real symbols which satisfy (2) so that $|b| \leq \sum\left|a_{j}\right|$. Then

$$
\left\|B^{w}\left(\frac{x+y}{2}, D\right) u\right\|_{L^{2}} \lesssim \sum\left\|A_{j}^{w}\left(\frac{x+y}{2}, D\right) u\right\|_{L^{2}}+\|u\|_{L^{2}} .
$$


Here and below it is not important whether we use the Weyl calculus or not, since the differences are negligible. Using a Littlewood-Paley decomposition we also get

Corollary 1.7. Let $a_{j}, b$ be real symbols satisfying (5), (6) so that $|b| \leq \sum\left|a_{j}\right|$. Then (10) holds.

and also

Corollary 1.8. Let $a_{j}, b \in C^{2} S^{1}$ be real symbols so that $|b| \leq \sum\left|a_{j}\right|$. Then

$$
\|B(x, D) u\|_{L^{2}} \lesssim \sum\left\|A_{j}(x, D) u\right\|_{L^{2}}+\|u\|_{L^{2}} .
$$

At the heart of this problem lies a division question: If $b, a_{j}$ are smooth and

$$
|b| \leq \sum\left|a_{j}\right|
$$

does this imply that there is a smooth decomposition

$$
b=\sum c_{j} a_{j} ?
$$

The answer to this question is unfortunately negative, which is what makes this problem nontrivial. One can see this easily by looking at the simple example

$$
|x y| \leq x^{2}+y^{2} \text {. }
$$

Instead our approach uses the FBI transform to reduce the problem to a rough decomposition:

Proposition 1.9. Let $b, a_{j}$ be functions satisfying

$$
|b| \leq \sum\left|a_{j}\right|, \quad\left|\nabla^{2} b\right|,\left|\nabla^{2} a_{j}\right| \leq 1 .
$$

Then there exist functions $c_{j}$ so that

$$
b=\sum c_{j} a_{j},
$$

and

$$
\left|c_{j}\right| \lesssim 1, \quad\left|a_{j} \nabla c_{j}\right| \lesssim \frac{\left|a_{j}\right|}{\left|\nabla a_{j}\right|+\left|a_{j}\right|^{\frac{1}{2}}}
$$

The expression $a_{j} \nabla c_{j}$ is well defined as a distribution by

$$
a_{j} \nabla c_{j}=\nabla\left(a_{j} c_{j}\right)-c_{j} \nabla a_{j} .
$$

One can also pose a similar question for one sided estimates. Suppose that the symbols $b, a_{j}$ satisfy

$$
b \leq \sum\left|a_{j}\right|
$$

Then we can ask whether the following estimate is true:

$$
<B u, u>\lesssim\|u\|_{L^{2}}^{2}+\|u\|_{L^{2}} \sum\left\|A_{j} u\right\|_{L^{2}} .
$$

If $a_{j}, b \in S^{1}$ then this follows from the classical Fefferman-Phong inequality. Here we prove a similar result for rough symbols. The main scale invariant result is 
Theorem 4. Let $\mu>0$ and $a_{j}, b$ be real symbols satisfying (2) so that $b \leq \sum\left|a_{j}\right|$. Then

$$
<B^{w}\left(\frac{x+y}{2}, D\right) u, u>\lesssim\|u\|_{L^{2}}^{2}+\|u\|_{L^{2}} \sum\left\|A_{j}^{w}\left(\frac{x+y}{2}, D\right) u\right\|_{L^{2}} .
$$

Using a Littlewood-Paley decomposition we also get

Corollary 1.10. Let $a_{j}, b$ be real symbols satisfying (5), (6) so that $b \leq \sum\left|a_{j}\right|$. Then (14) holds.

This further gives

Corollary 1.11. Let $a_{j}, b \in C^{2} S^{1}, a_{j}$ real, so that $b \leq \sum\left|a_{j}\right|$. Then

$$
<B(x, D) u, u>\lesssim\|u\|_{L^{2}}^{2}+\|u\|_{L^{2}} \sum\left\|A_{j}(x, D) u\right\|_{L^{2}} .
$$

The proof of these results uses the decomposition in Proposition 1.9 combined with an additional argument:

Proposition 1.12. Let $b, a_{j}$ be functions satisfying

$$
b \leq \sum\left|a_{j}\right|, \quad\left|\nabla^{2} b\right|,\left|\nabla^{2} a_{j}\right| \leq 1 .
$$

Then $b$ can be represented as $b=b_{0}+b_{1}$ where

$$
b_{0} \leq 0, \quad\left|b_{1}\right| \lesssim \sum\left|a_{j}\right|, \quad\left|\nabla^{2} b_{i}\right| \lesssim 1
$$

The structure of the paper is as follows. In the next section we introduce the FBI transform and show how to conjugate pseudodifferential operators with respect to it. The main result contains the error estimates for the conjugation. This essentially repeats arguments in [10], with the difference that the results here are somewhat more precise and are done for the Weyl calculus. In Section 3 we use the conjugation to obtain equivalent formulations of Theorems 2, 3, 4 and we show how these follow from Propositions 1.1, 1.9, 1.12. The dyadic estimates are assembled together in Section 4, where we conclude the proof of all the above corollaries. Section 5 contains the proof of all the representation results in Propositions 1.1, 1.9, 1.12. Finally, in the last two section we give some applications to local solvability and unique continuation for principally normal operators.

\section{The FBI TRANSFORM AND ERROR ESTIMATES}

The FBI transform of a temperate distribution $f$ is a holomorphic function in $C^{n}$ defined as

$$
(T f)(z)=2^{-\frac{n}{2}} \pi^{-\frac{3 n}{4}} \int_{5} e^{-\frac{1}{2}(z-y)^{2}} f(y) d y
$$


For additional information about the FBI transform we refer the reader to Delort's monograph [2]. To understand better how it works, consider the $L^{2}$ normalized function

$$
f_{x_{0}, \xi_{0}}(y)=\pi^{-\frac{n}{4}} e^{-\frac{1}{2}\left(y-x_{0}\right)^{2}} e^{i \lambda\left(y-x_{0}\right)}
$$

which is localized in a neighborhood of size 1 of $x_{0}$ and frequency localized in a neighborhood of size 1 of $\xi_{0}$. Due to the uncertainty principle this is the best one can do when trying to localize in both space and frequency. Then

$$
(T f)(z)=\pi^{-\frac{n}{2}} e^{\frac{1}{4}\left(z-x_{0}+i \xi_{0}\right)^{2}-\frac{1}{2}\left(z-x_{0}\right)^{2}}=\pi^{\frac{n}{4}} e^{-\frac{1}{4}\left|z-x_{0}+i \xi_{0}\right|^{2}} e^{\frac{1}{2}|\Im z|^{2}} e^{-i \frac{1}{2}\left(\Re z-x_{0}\right)\left(\Im z-\xi_{0}\right)} .
$$

Modulo the common factor $e^{\frac{1}{2}|\Im z|^{2}}$ this is concentrated in a neighborhood of size 1 of $x_{0}-i \xi_{0}$. Hence, it is natural to introduce the notation

$$
z=x-i \xi
$$

Like the Fourier transform, the FBI transform has good $L^{2}$ properties. Set

$$
\Phi(z)=e^{-\lambda \xi^{2}} .
$$

Then the operator $T_{\lambda}$ is an isometry from $L^{2}\left(R^{n}\right)$ onto the closed subspace of holomorphic functions in $L_{\Phi}^{2}\left(C^{n}\right)$. One inversion formula is provided by the adjoint operator:

$$
f(y)=2^{-\frac{n}{2}} \pi^{-\frac{3 n}{4}} \int \Phi(z) e^{-\frac{1}{2}(\bar{z}-y)^{2}}(T f)(z) d x d \xi .
$$

This is of course not the only possible inversion formula since the range of $T$ consists only of holomorphic functions.

Our main goal is to see how to conjugate pseudodifferential operators with respect to the FBI transform. Such a computation was already carried out in [10]. However, for our purposes here we need somewhat stronger results. This is easier to achieve using the Weyl calculus.

Let $a(x, \xi)$ be a compactly supported symbol. What we want is to determine the conjugate $\tilde{A}^{w}$ of $A^{w}$ with respect to $T$,

$$
T A^{w} \approx \tilde{A}^{w} T,
$$

modulo a small remainder. To get started observe that

$$
T y=(x+(\partial-i \xi)) T, \quad T D=\left(\xi+\frac{1}{i}(\partial-i \xi)\right) T,
$$

where $\partial$ stands for the complex differentiation with respect to the variable $z=x-i \xi$. We want to carry out all the analysis in complex variables, so it is more useful to rewrite this in the form

$$
T(y-i D)=z T, \quad T(y+i D)=[\bar{z}+2(\partial-i \xi)] T .
$$

Based on this, one can take a Taylor series expansion and obtain the formal asymptotics

$$
T A^{w} \approx \sum_{\alpha \leq \beta} 2^{|\beta|-|\alpha|} \frac{\partial^{\alpha} \bar{\partial}^{\beta} a(x, \xi)}{\alpha !(\beta-\alpha) !}(\partial-i \xi)^{\beta-\alpha} T .
$$


To gain a better understanding of the size of the terms in the above series it is useful to recall the following estimate proved in [10]:

Lemma 2.1. The following estimate holds:

$$
\left\|(\partial-i \xi)^{\alpha} T u\right\|_{L_{\phi}^{2}}=c_{\alpha}\|u\|_{L^{2}} .
$$

We want to make such asymptotics rigorous. Given $k>0$ define the partial sum

$$
\tilde{A}_{k}^{w}=\sum_{\alpha \leq \beta}^{|\alpha|+|\beta|<k} 2^{|\beta|-|\alpha|} \frac{\partial^{\alpha} \bar{\partial}^{\beta} a(x, \xi)}{\alpha !(\beta-\alpha) !}(\partial-i \xi)^{\beta-\alpha} T
$$

and the remainder

$$
R_{k}=T A^{w}-\tilde{A}_{k}^{w} T
$$

Then our main result is

Theorem 5. Assume that the symbol $a(x, \xi)$ satisfies

$$
\left|\partial_{x}^{\alpha} \partial_{\xi}^{\beta} a(x, \xi)\right| \leq c_{\alpha, \beta}, \quad|\alpha|+|\beta| \geq k
$$

Then

$$
\left\|R_{k}\right\|_{L^{2} \rightarrow L_{\phi}^{2}} \lesssim 1
$$

Two special cases are of interest to us, namely $k=2$ and $k=4$. For $k=2$ we get

$$
\tilde{A}_{2}^{w}=a+2 \bar{\partial} a(\partial-i \xi) .
$$

For $k=4$, on the other hand,

(20) $\tilde{A}_{4}^{w}=a+2 \bar{\partial} a(\partial-i \xi)+2 \bar{\partial}^{2} a(\partial-i \xi)^{2}+\frac{4}{3} \bar{\partial}^{3} a(\partial-i \xi)^{3}+\partial \bar{\partial} a+2 \partial \bar{\partial}^{2} a(\partial-i \xi)$.

Proof. The operator $T A^{w}$ has the form

$$
\left(T A^{w} f\right)(z)=c_{n} \int e^{-\frac{1}{2}(z-\tilde{y})^{2}} a\left(\frac{y+\tilde{y}}{2}, \eta\right) e^{i(\tilde{y}-y) \eta} f(y) d y d \eta .
$$

Introduce the new complex variable

$$
w=\frac{y+\tilde{y}}{2}-i \eta
$$

Then

$$
\left(T A^{w} u\right)(z)=c_{n} \int a(w) e^{\phi(z, w, y)} u(y) d w d \bar{w} d y
$$

where

$$
\begin{aligned}
\phi(z, w, y) & =-\frac{1}{2}(z+y-w-\bar{w})^{2}+\frac{1}{2}(\bar{w}-w)(\bar{w}+w-2 y) \\
& =\bar{w}(z-w)-\frac{1}{2}\left[(z+y-w)^{2}+w(w-2 y)\right]
\end{aligned}
$$


We claim that $\tilde{A}_{k}^{w}$ is the operator obtained from this by replacing $a(w)$ by its $k$-th Taylor polynomial at $z$,

$$
\left(\tilde{A}_{k}^{w} T u\right)(z)=\sum_{|\alpha|+|\beta|<k} \frac{\partial^{\alpha} \bar{\partial}^{\beta} a(z)}{\alpha ! \beta !} \int(w-z)^{\alpha}(\bar{w}-\bar{z})^{\beta} e^{\phi(z, w, y)} u(y) d w d \bar{w} d y .
$$

To prove this assertion we integrate by parts. Observe that

$$
\frac{\partial \phi}{\partial \bar{w}}=(z-w), \quad 2\left(\frac{\partial \phi}{\partial z}-i \xi\right)-\frac{\partial \phi}{\partial w}=(\bar{w}-\bar{z}) .
$$

Then we use the first relation to eliminate all the $(w-z)$ factors and the second relation to deal with the $(\bar{w}-\bar{z})$ factors. We get

$$
\begin{aligned}
\int(w-z)^{\alpha}(\bar{w}-\bar{z})^{\beta} e^{\phi(z, w, y)} d w d \bar{w} & =\frac{\beta !}{(\beta-\alpha) !} \int(\bar{w}-\bar{z})^{\beta-\alpha} e^{\phi(z, w, y)} d w d \bar{w} \\
& =\frac{\beta !}{(\beta-\alpha) !}\left[2\left(\frac{\partial}{\partial z}-i \xi\right)\right]^{\beta-\alpha} \int e^{\phi(z, w, y)} d w d \bar{w} .
\end{aligned}
$$

This proves (21). Note that the above computations are rigorous since the phase function $\phi$ is non-positive definite.

It remains to prove the $L^{2} \rightarrow L_{\phi}^{2}$ remainder bound. It is easier to visualize the analysis in the real setting, where $R_{k}$ has the form

$$
\left(R_{k} u\right)(x, \xi)=\int b\left(x, \xi, \frac{y+\tilde{y}}{2}, \eta\right) e^{-\frac{1}{2}(x-i \xi-y)^{2}} e^{i(\tilde{y}-y) \eta} d \tilde{y} d \eta .
$$

and $b$ is the order $k$ remainder in the Taylor series for the symbol $a$,

$$
b(x, \xi, y, \eta)=a(y, \eta)-\sum_{|\alpha|+|\beta|<k} \frac{\partial_{x}^{\alpha} \partial_{\xi}^{\beta} a(x, \xi)}{\alpha ! \beta !}(y-x)^{\alpha}(\xi-\eta)^{\beta} .
$$

Note that $b$ satisfies the bounds

$$
\left|\partial_{y}^{\alpha} \partial_{\eta}^{\beta} b(x, \xi, y, \eta)\right| \leq c_{\alpha, \beta}(|x-y|+|\xi-\eta|)^{\max \{k-|\alpha|-|\beta|, 0\}} .
$$

We can eliminate part of the exponential weight if we observe that

$$
\left\|R_{k}\right\|_{L^{2} \rightarrow L_{\phi}^{2}}=\left\|e^{-i x \xi} e^{-\frac{1}{2} \xi^{2}} R_{k}\right\|_{L^{2} \rightarrow L^{2}} .
$$

We write the kernel $H(x, \xi, y)$ of

$$
R=e^{-i x \xi} e^{-\frac{1}{2} \xi^{2}} R_{k}
$$

in the form

$$
H(x, \xi, y)=e^{-i \xi y} c(x, \xi, y-x)
$$

where

$$
c(x, \xi, y-x)=\int b\left(x, \xi, \frac{y+\tilde{y}}{2}, \eta\right) e^{-\frac{1}{2}(x-\tilde{y})^{2}} e^{i(\tilde{y}-y)(\eta-\xi)} d \tilde{y} d \eta .
$$

After the change of variable $\eta:=\eta-\xi, \tilde{y}:=\tilde{y}-x$ this becomes

$$
c(x, \xi, y)=\int b\left(x, \xi, x+\frac{y+\tilde{y}}{2}, \xi+\eta\right) e^{-\frac{1}{2} \tilde{y}^{2}} e^{i(\tilde{y}-y) \eta} d \tilde{y} d \eta .
$$


We claim that $c$ is a Schwartz function in $y$, uniformly in $x, \xi$. Given the bound (22) on $b$, it suffices to show that the function

$$
c(y)=\int b(y+\tilde{y}, \eta) e^{-\frac{1}{2} \tilde{y}^{2}} e^{i(\tilde{y}-y) \eta} d \tilde{y} d \eta
$$

is a Schwartz function provided that $b$ satisfies

$$
\left|\partial_{y}^{\alpha} \partial_{\eta}^{\beta} b(y, \eta)\right| \leq c_{\alpha, \beta}(|y|+|\eta|)^{\max \{k-|\alpha|-|\beta|, 0\}} .
$$

Indeed, the function $b(y+\tilde{y}, \eta) e^{-\frac{1}{2} \tilde{y}^{2}}$ is a Schwartz function in $\tilde{y}$ of size at most $(|y|+|\eta|)^{k}$. Then integrating with respect to $\tilde{y}$ we get a Schwartz function in $\eta$ of size at most $|y|^{k}$. Finally, integrating with respect to $\eta$ we get a Schwartz function in $y$.

The $L^{2} \rightarrow L^{2}$ boundedness of $R$ is equivalent to the $L^{2}$ boundedness of $R R^{*}$, whose kernel is

$$
K(x, \xi, \tilde{x}, \tilde{\xi})=\int c(x, \xi, x-y) c(\tilde{x}, \tilde{\xi}, \tilde{x}-y) e^{i y(\tilde{\xi}-\xi)} d \eta
$$

Integrating with respect to $y$ we get

$$
|K(x, \xi, \tilde{x}, \tilde{\xi})| \leq c_{N}(1+|x-\tilde{x}|+|\xi-\tilde{\xi}|)^{-N}
$$

and the $L^{2}$ boundedness follows.

\section{The DYADIC RESUlts}

The aim of this section is to prove Theorems 2, 3, 4 using the representation results in Propositions 1.1, 1.9, 1.12. We can rescale and assume that $\mu=1$. We start with the counterpart of the sharp Garding's inequality, namely Theorem 2(a). This is quite easy and it illustrates well one aspect of the more involved computations later on.

Proof of Theorem 2,(a). By Theorem 5 with $k=2$, the semipositivity of $A^{w}$ is equivalent to the semipositivity of $\tilde{A}_{2}^{w}$ on the space of holomorphic functions in $L_{\Phi}^{2}$,

$$
<\tilde{A}_{2}^{w} v, v>\geq-c\|v\|_{L_{\Phi}^{2}}^{2}
$$

where $v=T u$. The operator $\tilde{A}_{2}^{w}$ is given by (19). The useful observation is that $(\partial-i \lambda \xi)$ is the adjoint operator to $-\bar{\partial}$ in $L_{\Phi}^{2}$. Since $v$ is holomorphic, this implies that

$$
\begin{aligned}
<\tilde{A}_{\lambda, 2}^{w} v, v> & =<(a+2 \bar{\partial} a(\partial-i \lambda \xi)) v, v>_{\Phi} \\
& =<(a-2 \partial \bar{\partial} a) v, v>_{\Phi} .
\end{aligned}
$$

Then the conclusion follows since $a$ is nonnegative and $|\partial \bar{\partial} a| \lesssim 1$.

Proof of Theorem 2,(b). By Theorem 5 with $k=4$, the semipositivity of $A^{w}$ is equivalent to the semipositivity of $\tilde{A}_{4}^{w}$ on the space of holomorphic functions in $L_{\Phi}^{2}$,

$$
<\tilde{A}_{4}^{w} v, v>_{\Phi} \geq-c\|v\|_{L_{\Phi}^{2}}^{2}
$$

where $v=T u$. At this point we discard all the information about the higher order derivatives of $a$ and only retain the bound on the fourth order derivatives. The 
operator $\tilde{A}_{4}^{w}$ is given by (20). Then, as before, we integrate by parts all the terms in $\tilde{A}_{\lambda, 4}^{w}$ which contain $(\partial-i \lambda \xi)$,

$$
<2 \bar{\partial} a(\partial-i \xi) v, v>_{\Phi}=-2<(\partial \bar{\partial} a) v, v>_{\Phi} .
$$

This term in not necessarily bounded in $L_{\phi}^{2}$ since we have no information about the second derivatives of $a$.

$$
<2 \bar{\partial}^{2} a(\partial-i \xi)^{2} v, v>_{\Phi}=2<\left(\partial^{2} \bar{\partial}^{2} a\right) v, v>_{\Phi} .
$$

This is bounded since the fourth derivatives of $a$ are bounded.

$$
<\frac{4}{3} \bar{\partial}^{3} a(\partial-i \xi)^{3} v, v>_{\Phi}=-\frac{4}{3}<\left(\partial \bar{\partial}^{3} a\right)(\partial-i \xi)^{2} v, v>
$$

This is also bounded by Lemma 2.1 .

$$
<2 \partial \bar{\partial}^{2} a(\partial-i \xi) v, v>_{\Phi}=-2<\left(\partial^{2} \bar{\partial}^{2} a\right) v, v>_{\Phi}
$$

which is also bounded. Summing up all these estimates we obtain the following simple formula:

$$
<\tilde{A}_{4}^{w} v, v>_{\Phi}=<(a-\partial \bar{\partial} a) v, v>_{\Phi}+O\left(\|v\|_{L_{\Phi}^{2}}^{2}\right) .
$$

By Proposition 1.1 we can assume without any restriction in generality that $a=\phi^{2}$ where $\phi$ satisfies the bounds in (4). Then

$$
a-\partial \bar{\partial} a=\phi^{2}-2(\partial \phi)(\bar{\partial} \phi)-2 \phi(\partial \bar{\partial} \phi) .
$$

On the other hand we use the same integration by parts procedure to evaluate the nonnegative quadratic form

$$
\begin{aligned}
Q(v, v)= & \|(\phi+2 \bar{\partial} \phi(\partial-i \xi)+\partial \bar{\partial} \phi) v\|_{L_{\Psi}^{2}}^{2} \\
= & \left.<(\phi+\partial \bar{\partial} \phi)^{2} v, v>_{\Phi}+4 \Re<(\partial \phi)(\phi+\partial \bar{\partial} \phi) v,(\partial-i \xi)\right) v>_{\Phi} \\
+ & 4<\bar{\partial} \phi(\partial-i \xi)) v, \bar{\partial} \phi(\partial-i \xi)) v>_{\Phi} \\
= & <(\phi+\partial \bar{\partial} \phi)^{2} v, v>_{\Phi}-4 \Re<[\bar{\partial}((\partial \phi)(\phi+\partial \bar{\partial} \phi))] v, v>_{\Phi} \\
+ & 4<\frac{1}{2}(\partial \phi)(\bar{\partial} \phi)+\partial \bar{\partial}(\partial \phi)(\bar{\partial} \phi) v, v>_{\Phi} \\
= & <\left[\phi^{2}-2(\partial \phi)(\bar{\partial} \phi)-2 \phi(\partial \bar{\partial} \phi)\right] v, v>_{\Phi} \\
+ & <\left[(\partial \bar{\partial} \phi)^{2}-4 \Re \bar{\partial}(\partial \phi)(\partial \bar{\partial} \phi)+4 \partial \bar{\partial}(\partial \phi)(\bar{\partial} \phi)\right] v, v>_{\Phi}^{2} .
\end{aligned}
$$

By (4) the second term is bounded, therefore

$$
<\tilde{A}_{4}^{w} v, v>_{\Phi}=Q(v, v)+O\left(\|v\|_{L_{\Phi}^{2}}^{2}\right) .
$$

Hence the semipositivity of $\tilde{A}_{\lambda, 4}^{w}$ follows.

Proof of Theorem 3. Set $v=T u$. Then according to the conjugation result in Theorem 5 ,

$$
\left\|A_{j}^{w} u\right\|_{L^{2}}=\left\|\tilde{A}_{j, 2}^{w} v\right\|_{L_{\Phi}^{2}}+O\left(\|v\|_{L_{\Phi}^{2}}\right) .
$$


and similarly for $B$. Hence we need to show that

$$
\left\|\tilde{B}_{2}^{w} v\right\|_{L_{\Phi}^{2}} \lesssim \sum\left\|\tilde{A}_{j, 2}^{w} v\right\|_{L_{\Phi}^{2}}+\|v\|_{L^{2}} .
$$

We use the decomposition in Proposition 3 to evaluate

$$
\begin{aligned}
\left\|\tilde{B}_{2}^{w} v\right\|_{L_{\Phi}^{2}} & =\|(b+2(\bar{\partial} b)(\partial-i \xi)) v\|_{L_{\Phi}^{2}} \\
& =\left\|\left(\sum c_{j} a_{j}+2\left(\bar{\partial} c_{j} a_{j}\right)(\partial-i \xi)\right) v\right\|_{L_{\Phi}^{2}} \\
& \left.\lesssim \sum\left\|\tilde{A}_{j, 2}^{w} v\right\|_{L_{\Phi}^{2}}+\| a_{j}\left(\bar{\partial} c_{j}\right)(\partial-i \xi)\right) v \|_{L_{\Phi}^{2}} \\
& \left.\lesssim \sum\left\|\tilde{A}_{j, 2}^{w} v\right\|_{L_{\Phi}^{2}}+\| \frac{a_{j}}{\left(\left|\nabla a_{j}\right|^{2}+\left|a_{j}\right|\right)^{\frac{1}{2}}}(\partial-i \xi)\right) v \|_{L_{\Phi}^{2}}
\end{aligned}
$$

Then it remains to bound the second right hand side term. The function

$$
d_{j}=\frac{a_{j}}{\left(\left|\nabla a_{j}\right|^{2}+\left|a_{j}\right|\right)^{\frac{1}{2}}}
$$

is Lipschitz, therefore, integrating by parts,

$$
\begin{aligned}
\left\|d_{j}(\partial-i \xi) v\right\|_{L_{\Phi}^{2}} & \lesssim\left\|(\partial-i \xi) d_{j} v\right\|_{L_{\Phi}^{2}}+\|v\|_{L_{\Phi}^{2}} \\
& \lesssim\left\|d_{j} v\right\|_{L_{\Phi}^{2}}+\left\|\bar{\partial} d_{j} v\right\|_{L_{\Phi}^{2}}+\|v\|_{L_{\Phi}^{2}} \\
& \lesssim\left\|d_{j} v\right\|_{L_{\Phi}^{2}}+\|v\|_{L_{\Phi}^{2}} .
\end{aligned}
$$

It remains to show that

$$
\left\|d_{j} v\right\|_{L_{\Phi}^{2}} \lesssim\left\|\left(a_{j}+2\left(\bar{\partial} a_{j}\right)(\partial-i \xi)\right) v\right\|_{L_{\Phi}^{2}}+\|v\|_{L^{2}} .
$$

This is a consequence of the following computation:

$<\left(a_{j}+2\left(\bar{\partial} a_{j}\right)(\partial-i \xi)\right) v, \frac{a_{j}}{\left|\nabla a_{j}\right|^{2}+\left|a_{j}\right|} v>_{\Phi}=\left\|d_{j} v\right\|_{L_{\Phi}^{2}}^{2}-2<\left[\bar{\partial}\left(\frac{a_{j} \partial a_{j}}{\left|\nabla a_{j}\right|^{2}+\left|a_{j}\right|}\right)\right] v, v>_{\Phi}$

The second right hand side term is bounded because the function $\frac{a_{j} \partial a_{j}}{\left|\nabla a_{j}\right|^{2}+\left|a_{j}\right|}$ is Lipschitz. This follows easily since the second derivatives of $a_{j}$ are bounded.

Proof of Theorem 4. Rescale to $\mu=1$. Using the conjugation result in Theorem 5, the estimate (13) is equivalent to

$$
<\tilde{B}_{2}^{w} v, v>_{\Phi} \lesssim \sum\left\|\tilde{A}_{j, 2}^{w} v\right\|_{L_{\Phi}^{2}}+\|v\|_{L^{2}}
$$

From this point on we only use the information about the second order derivatives of the symbols $b, a_{j}$. We use Proposition 1.12 to decompose $b=b_{0}+b_{1}$. Then we treat $b_{0}$ as in the proof of Theorem 2(a) (the sharp Garding inequality) and $b_{1}$ as in the proof of Theorem 3. 


\section{Littlewood-Paley decompositions and Symbol Smoothing}

In this section we obtain Corollaries 1.2-1.6 from Theorem 2 and Corollary 1.7 from Theorem 3. These are all routine arguments therefore we leave the reader to fill in the details where necessary. For the Weyl calculus we refer the reader to [8], and for the symbol smoothing, to [11].

Proof of Corollary 1.2. We start with a smooth function $s(\xi)$ supported in $\frac{1}{2} \leq|\xi| \leq 2$ so that

$$
1=s_{0}(\xi) \sum_{\lambda \geq 1, \text { dyadic }} s_{\lambda}(\xi), \quad s_{\lambda}(\xi)=s\left(\frac{\xi}{\lambda}\right) .
$$

Correspondingly we decompose

$$
a(x, \xi)=\sum a_{\lambda}(x, \xi), \quad a_{\lambda}(x, \xi)=s_{\lambda}(\xi) a(x, \xi) .
$$

If the symbol $a$ satisfies (5) and (6) then $a_{\lambda}$ satisfies (2) with $\mu=\lambda^{\rho}$ so we can apply Theorem 2(a). There is, however, the question of summation with respect to $\lambda$. To deal with that we introduce the additional operators

$$
T_{\lambda}=\sum_{|j| \leq 4} S_{2^{j} \lambda}
$$

Then

$$
A_{\lambda}^{w}=T_{\lambda} A_{\lambda}^{w} T_{\lambda}+R_{\lambda},
$$

where the remainders $R_{\lambda}$ are rapidly decaying in $\lambda$ because the symbol of $T_{\lambda}$ equals 1 in a neighborhood of the support of $a_{\lambda}$. Then by Theorem 2(a) we get

$$
\begin{aligned}
<A^{w} u, u> & =\sum_{\lambda \geq 1, \text { dyadic }}<A_{\lambda}^{w} T_{\lambda} u, T_{\lambda} u>+O\left(\|u\|_{L^{2}}\right) \\
& \geq-c \sum\left\|T_{\lambda} u\right\|^{2}+O\left(\|u\|_{L^{2}}\right) \\
& =O\left(\|u\|_{L^{2}}^{2}\right) .
\end{aligned}
$$

Similarly, if the symbol $a$ satisfies (7) and (8) then $a_{\lambda}$ satisfies (3) so we can apply Theorem 2(b) instead.

Proof of Corollaries 1.3-1.6. The next result allows us to replace the Weyl operators $A^{w}\left(\frac{x+y}{2}, D\right)$ with $A(x, D)$ or $A(D, y)$ in Corollary 1.2.

Lemma 4.1. a) Let $0 \leq \rho<1$ and a be a symbol satisfying (5). Then

$$
a^{w}\left(\frac{x+y}{2}, D\right)-a(x, D) \in O P S_{\rho, \rho}^{0},
$$

therefore it is $L^{2}$ bounded.

b) Let a be a symbol satisfying (7). Then

$$
A^{w}\left(\frac{x+y}{2}, D\right)-\frac{a(x, D)+A(D, y)}{2} \in O P S_{\rho, \rho}^{0}
$$

therefore it is $L^{2}$ bounded. 
The proof is straightforward. Taking this into account, Corollaries 1.3-1.6 follow from the following symbol smoothing argument:

Lemma 4.2. Let $s>0, k$ a nonnegative integer and $a(x, \xi) \in C^{s} S^{\frac{k s}{k+s}}$. Then there is a decomposition

$$
a(x, \xi)=a_{0}(x, \xi)+r(x, \xi),
$$

where $a_{0}$ satisfies

$$
\begin{aligned}
& \qquad \partial_{x}^{\alpha} \partial_{\xi}^{\beta} a_{0}(x, \xi)\left|\leq c_{\alpha, \beta}(1+|\xi|)^{\frac{k}{k+s}(|\alpha|-|\beta|)}, \quad\right| \alpha|+| \beta \mid \geq k \\
& \\
& \left|\partial_{x}^{\alpha} \partial_{\xi}^{\beta} a_{0}(x, \xi)\right| \leq c_{\alpha, \beta}(1+|\xi|)^{\frac{k s}{k+s}+\frac{k-s}{k+s}|\alpha|-|\beta|}, \quad|\alpha|+|\beta|<k \\
& \text { and } r(x, \xi) \in C^{s} S_{1, \frac{k}{k+s}}^{0} .
\end{aligned}
$$

This is done as in [11]. Roughly speaking, $a_{0}$ is obtained by regularizing $a$ in $x$ on the $\lambda^{-\frac{k}{k+s}}$ scale for $|\xi|=O(\lambda)$.

The remainder $R$ is $L^{2}$ bounded, so the Lemma allows us to use Corollary 1.2(a) if $k=2$, respectively Corollary $1.2(\mathrm{~b})$ if $k=4$. In both cases $\rho=\frac{k}{k+s}$.

Proof of Corollary 1.7. With the same notations as in the proof of Corollary 1.2 we define the frequency localized symbols $a_{j, \lambda}, b_{\lambda}$. These will satisfy the conditions in Theorem 3 , therefore applying it to $T_{\lambda} u$ we get

$$
\left\|B_{\lambda}^{w} T_{\lambda} u\right\|_{L^{2}} \lesssim \sum\left\|A_{j, \lambda}^{w} T_{\lambda} u\right\|_{L^{2}}+\left\|T_{\lambda} u\right\|_{L^{2}} .
$$

We square this and sum up. To get (10) we need to verify that

$$
\left\|B^{w} u\right\|_{L^{2}}^{2} \approx \sum_{\lambda \text { dyadic }}\left\|B_{\lambda}^{w} T_{\lambda} u\right\|_{L^{2}}^{2}+O\left(\|u\|_{L^{2}}^{2}\right)
$$

and similarly for $A_{j}^{w}$. Indeed, we have

$$
\left\|B^{w} u\right\|_{L^{2}}^{2} \approx \sum\left\|S_{\lambda} B^{w} u\right\|_{L^{2}}^{2} .
$$

Since the symbol of $T_{\lambda}$ is 1 in a $\lambda$ neighborhood of the support of $s_{\lambda}$, it follows that the difference $S_{\lambda} B^{w} T_{\lambda}-S_{\lambda} B^{w}$ is rapidly decreasing in $\lambda$. Hence we get

$$
\left\|B^{w} u\right\|_{L^{2}}^{2} \approx \sum\left\|S_{\lambda} B^{w} T_{\lambda} u\right\|_{L^{2}}^{2}+O\left(\|u\|_{L^{2}}^{2}\right) .
$$

Then (25) follows since the operators $S_{\lambda} B^{w}-B_{\lambda}^{w}$ are uniformly bounded in $L^{2}$.

Proof of Corollary 1.8. First we use the symbol regularization in Lemma 4.2 with $k=s=2$ to reduce the problem to the case when the symbols $b, a_{j}$ satisfy the conditions (5) and (6). Since the difference between the original and the regularized symbols is bounded, we still have

$$
|b| \lesssim 1+\sum\left|a_{j}\right|
$$

Then observe that Lemma 4.1 allows us to interchange the operators $B(x, D)$ and $B^{w}$ and reduce the estimate to the one in Corollary 1.7. 
Proof of Corollary 1.10. As above, one uses a Littlewood-Paley decomposition to reduce the problem to the dyadic estimates in Theorem 4 . The operator $B$ is dealt with as in the proof of Corollary 1.2(a), while for the operators $A_{j}$ one argues as in the proof of Corollary 1.7.

Proof of Corollary 1.11. This follows from Corollary 1.10) after using the symbol regularization in Lemma 4.2 combined with Lemma 4.1.

\section{REPRESENTATIONS OF FUNCTIONS}

Proof of Proposition 1.1. The proof consists entirely of classical arguments. However, the simplicity of the result makes it worthwhile to present a complete argument. We use induction with respect to the space dimension $n$. We begin the induction at $n=0$, where any nonnegative number is the square of another real number.

Observe that the result is invariant with respect to the scaling

$$
\phi(x) \rightarrow \lambda^{-4} \phi(\lambda x), \quad \phi_{k}(x) \rightarrow \lambda^{-2} \phi_{k}(\lambda x) .
$$

This is used repeatedly in order to simplify the arguments below.

We define

$$
\left|\nabla^{j} \phi(x)\right|=\sup _{\left|u_{k}\right| \leq 1}\left|\left(D^{j} \phi(x)\right)\left(u_{1}, \cdots, u_{j}\right)\right| .
$$

For the gradient this gives the euclidean norm, for the Hessian, the largest eigenvalue. Such precise definitions are only necessary in order to perform the explicit computations in the sequel. It is easy to verify that the following relations hold:

$$
\left|\nabla^{2} \phi\right|=\sup _{|u| \leq 1}\left|\left(D^{2} \phi\right)(u, u)\right| \quad\left|\nabla^{3} \phi\right| \leq 2 \sup _{|u| \leq 1}\left|\left(D^{3} \phi\right)(u, u, u)\right| .
$$

We start with a bound for the odd derivatives of $\phi$ in terms of the even ones.

Lemma 5.1. Let $\phi$ be a nonnegative function in $\mathbb{R}^{n}$ satisfying

$$
\left|\nabla^{4} \phi\right| \leq 1
$$

Then

$$
\begin{gathered}
|\nabla \phi(x)| \leq 2 \phi(x)^{\frac{3}{4}}+3 \phi(x)^{\frac{1}{2}}\left|\nabla^{2} \phi(x)\right|^{\frac{1}{2}} \\
\left|\nabla^{3} \phi(x)\right| \leq 8 \phi(x)^{\frac{1}{4}}+8\left|\nabla^{2} \phi(x)\right|^{\frac{1}{2}} .
\end{gathered}
$$

Proof. Without any restriction in generality we assume that $x=0$. By (26) we can also assume that $n=1$ at the expense of replacing 8 by 4 in (28). Write the Taylor series at 0 for $\phi$,

$$
\phi(y) \leq \phi(0)+y \phi^{\prime}(0)+\frac{1}{2} y^{2} \phi^{\prime \prime}(0)+\frac{1}{6} y^{3} \phi^{\prime \prime \prime}(0)+\frac{1}{24} y^{4} .
$$

If we use the nonnegativity condition for $\phi$ at $\pm y$ then we obtain

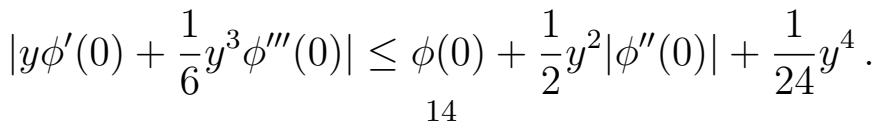


Substituting $y$ by $2 y$ yields

$$
\left|2 y \phi^{\prime}(0)+\frac{4}{3} y^{3} \phi^{\prime \prime \prime}(0)\right| \leq \phi(0)+2 y^{2}\left|\phi^{\prime \prime}(0)\right|+\frac{2}{3} y^{4} .
$$

If we combine these two bounds we get

$$
\begin{gathered}
\left|y \phi^{\prime}(0)\right| \leq \frac{3}{2} \phi(0)+y^{2}\left|\phi^{\prime \prime}(0)\right|+\frac{1}{6} y^{4} . \\
\left|y^{3} \phi^{\prime \prime \prime}(0)\right| \leq 3 \phi(0)+3 y^{2}\left|\phi^{\prime \prime}(0)\right|+y^{4} .
\end{gathered}
$$

Then (27) follows if we set

$$
y=\frac{\phi(0)^{\frac{1}{2}}}{\phi(0)^{\frac{1}{4}}+\phi^{\prime \prime}(0)^{\frac{1}{2}}} .
$$

in the first relation, while (28) follows from the second relation with

$$
y=\max \left\{\phi(0)^{\frac{1}{4}}, \phi^{\prime \prime}(0)^{\frac{1}{2}}\right\} .
$$

The next step is to define the function

$$
r(x)=\max \left\{\left|\nabla^{2} \phi(x)\right|^{\frac{1}{2}},|\phi(x)|^{\frac{1}{4}}\right\},
$$

which shall be used to measure the size of $\phi$ near $x$. An essential property of $r$ is that it is "slowly varying", i.e.

Lemma 5.2. Let $\phi$ be a nonnegative function in $\mathbb{R}^{n}$ satisfying $\left|\nabla^{4} \phi\right| \leq 1$ and $r$ defined as above. Then

$$
|r(x)-r(y)| \leq \frac{1}{2} r(x) \quad \text { for } \quad|x-y| \leq \frac{1}{50} r(x) .
$$

Proof. By translation and rescaling we can assume that $x=0$ and $r(x)=1$. Then

$$
\phi(0) \leq 1, \quad|\nabla \phi(0)| \leq 5, \quad\left|\nabla^{2} \phi(0)\right| \leq 1, \quad\left|\nabla^{3} \phi(0)\right| \leq 16,
$$

therefore, for $|y-x| \leq \frac{1}{50}$, Taylor's formula gives

$$
|\phi(y)-\phi(0)| \leq \frac{5}{50}+\frac{1}{2 \cdot 50^{2}}+\frac{4}{3 \cdot 50^{3}}+\frac{1}{50^{4}} \leq \frac{1}{2}
$$

while

$$
\left|\nabla^{2} \phi(y)-\nabla^{2} \phi(0)\right| \leq \frac{16}{50}+\frac{1}{2 \cdot 50^{2}} \leq \frac{1}{2} .
$$

Given the definition of $r$, the last two relations imply the desired conclusion.

As an immediate consequence of the previous Lemma we get

Corollary 5.3. Let $\phi$ be a nonnegative function in $\mathbb{R}^{n}$ satisfying $\left|\nabla^{4} \phi\right| \leq 1$ and $r$ defined as above. Then

$$
B\left(x, \frac{1}{50} r(x)\right) \cap B\left(y, \frac{1}{50} r(y)\right) \neq \emptyset \quad \Longrightarrow \quad \frac{1}{3} r(x) \leq r(y) \leq 3 r(x) .
$$


Proof. Let $z \in B\left(x, \frac{1}{50} r(x)\right) \cap B\left(y, \frac{1}{50} r(y)\right)$. Then by Lemma 5.2 we have

$$
\frac{1}{2} r(x) \leq r(z) \leq \frac{3}{2} r(x), \quad \frac{1}{2} r(y) \leq r(z) \leq \frac{3}{2} r(y),
$$

therefore the conclusion follows.

The next step in the proof is a Whitney type covering lemma. The goal is to obtain a partition of unit which allows us to isolate the "building blocks" of the function $\phi$.

Lemma 5.4. Let $0<\epsilon \leq \frac{1}{50}$ be a small parameter. There exists a sequence $\left\{x_{k}\right\}$ in $\mathbb{R}^{n}$ and a partition of unit

$$
1=\sum \chi_{k}^{2}(x) \quad \text { in } \quad\{r(x)>0\}
$$

with the following properties:

i) The support of $\chi_{k}$ is contained in $B\left(x_{k}, \epsilon r\left(x_{k}\right)\right)$.

ii) There is some $N$ depending only on $n$ so that each ball $B\left(x_{k}, \epsilon r\left(x_{k}\right)\right)$ intersects at most $N$ others.

iii) $\left|\nabla^{\alpha} \chi_{k}\right| \leq c_{\alpha}\left(\epsilon r\left(x_{k}\right)\right)^{-\alpha}$, where $c_{\alpha}$ depends only on $\alpha$ and $n$.

Proof. Start with a maximal collection $x_{k}$ so that the balls $B\left(x_{k}, \frac{\epsilon}{8} r\left(x_{k}\right)\right)$ are disjoint. Our first claim is that

$$
\bigcup_{k} B\left(x_{k}, \frac{\epsilon}{2} r\left(x_{k}\right)\right)=\left\{x \in \mathbb{R}^{n} ; r(x) \neq 0\right\} .
$$

The " $\subset$ " part follows from Lemma 5.2. To obtain the " $\supset$ " inclusion let $x$ be so that $r(x)>0$. Then there exists some $k$ so that

$$
B\left(x, \frac{\epsilon}{8} r(x)\right) \cap B\left(x_{k}, \frac{\epsilon}{8} r\left(x_{k}\right)\right) \neq \emptyset .
$$

By Corollary 5.3 this implies that

$$
r(x) \leq 3 r\left(x_{k}\right)
$$

which further gives $x \in B\left(x_{k}, \frac{\epsilon}{2} r\left(x_{k}\right)\right)$ and concludes the proof of (29).

Corollary 5.3 also shows that if two balls $B\left(x_{k}, \operatorname{\epsilon r}\left(x_{k}\right)\right)$ intersect then they must have comparable radiuses. But on the other hand the balls $B\left(x_{k}, \frac{\epsilon}{6} r\left(x_{k}\right)\right)$ are disjoint, therefore a ball $B\left(x_{k}, \epsilon r\left(x_{k}\right)\right)$ can only intersect finitely many others.

Thus we have obtained a family of balls satisfying (i) and (ii). It remains to construct the corresponding partition of unit. This can be done in a standard fashion. We start with a smooth function $\chi$ supported in $B(0,1)$ and which is 1 in $B\left(0, \frac{1}{2}\right)$ and define

$$
\chi_{k}=\chi\left(\frac{x-x_{k}}{\epsilon r\left(x_{k}\right)}\right)\left(\sum_{j} \chi^{2}\left(\frac{x-x_{j}}{\epsilon r\left(x_{j}\right)}\right)\right)^{-\frac{1}{2}} .
$$

It is easy to see that this satisfies (iii). 
We now continue the proof of Proposition 1.1. Due to the finite intersection property for their supports, it suffices to express each of the the functions $\chi_{k}^{2} \phi$ as sums of squares which satisfy uniformly the bounds (4). Here it is important that the number of such squares depends only on the dimension; then one can group them together into finitely many sums of squares with disjoint supports.

After translation and rescaling we can assume without any restriction in generality that $x_{k}=0$ and $r(0)=1$. Then by Lemmas 5.1,5.2 it follows that $\phi$ and all its derivatives up to order 4 are bounded in the ball $B(0,1 / 50)$,

$$
|\phi| \leq \frac{3}{2}, \quad|\nabla \phi| \leq 8, \quad\left|\nabla^{2} \phi\right| \leq \frac{3}{2}, \quad\left|\nabla^{3} \phi\right| \leq 24, \quad\left|\nabla^{4} \phi\right| \leq 1 .
$$

In what follows we choose $\epsilon$ to be sufficiently small but fixed; $\epsilon=10^{-8}$ is sufficiently small. By $c(\epsilon)$ we denote various constants which depend only on $\epsilon$. Once $\epsilon$ is fixed these are absolute constants. We consider two cases:

I. $\phi(0) \geq 10 \epsilon$. Then for $x \in B(0, \epsilon)$ and $\epsilon \leq \frac{1}{50}$ we have

$$
\phi(x) \geq 10 \epsilon-5 \epsilon-\frac{1}{2} \epsilon^{2}-8 \frac{1}{6} \epsilon^{3}-\frac{1}{24} \epsilon^{4} \geq 4 \epsilon .
$$

Hence we simply define the function $\phi_{k}$ as

$$
\phi_{k}=\chi_{k} \phi^{\frac{1}{2}} \text {. }
$$

It is of class $C^{3,1}$ Since $\phi$ stays away from 0 in the support of $\chi_{k}$ it follows that $\phi_{k}$ is of class $C^{3,1}$,

$$
\left|\nabla^{\alpha} \phi_{k}\right| \leq c(\epsilon), \quad|\alpha| \leq 4
$$

II. $\phi\left(x_{k}\right) \leq 10 \epsilon$. Then $\left|\nabla^{2} \phi(0)\right|=1$. After a rotation we can assume that $\partial_{x_{1}}^{2}(0)=$ 1. Then we can use (30) to get

$$
\partial_{x_{1}}^{2} \phi(x)>\frac{1}{2} \quad x \in B\left(0, \frac{1}{50}\right) .
$$

On the other hand, (30) also shows that

$$
\phi(x)<26 \epsilon \quad x \in B(0,2 \epsilon) .
$$

Then by Lemma 5.1 we obtain

$$
\left|\partial_{x_{1}} \phi(x)\right| \leq 50 \sqrt{\epsilon}, \quad x \in B(0,2 \epsilon) .
$$

Split the coordinates $x=\left(x_{1}, x^{\prime}\right)$ and consider the function $\phi\left(x_{1}, x^{\prime}\right)$ as a function of $x_{1}$ in the range

$$
D=\left\{\left|x^{\prime}\right| \leq 2 \epsilon,\left|x_{1}\right| \leq \frac{1}{100}\right\} .
$$

By (31), (32) it follows that $\phi\left(x_{1}, x^{\prime}\right)$ has a unique minimum point $X\left(x^{\prime}\right)$ in $D$ as a function of $x_{1}$, satisfying

$$
\left|X\left(x^{\prime}\right)\right| \leq 100 \sqrt{\epsilon} .
$$

The function $X\left(x^{\prime}\right)$ is given by the equation

$$
\left(\partial_{x_{1}} \phi\right)\left(X\left(x^{\prime}\right), x^{\prime}\right)=0 .
$$


By (30) $\partial_{x_{1}} \phi$ is of class $C^{2,1}$ in $D$. By its level sets are nondegenerate. It follows that $X\left(x^{\prime}\right)$ is of class $C^{2,1}$ as well,

$$
\left|\nabla^{j} X\left(x^{\prime}\right)\right| \leq c(\epsilon), \quad j=0,1,2,3 \quad\left|x^{\prime}\right| \leq 2 \epsilon .
$$

Define the function

$$
\phi_{1}\left(x^{\prime}\right)=\phi\left(X\left(x^{\prime}\right), x^{\prime}\right) .
$$

Then we can decompose the function $\phi$ within $D$ as

$$
\phi(x)=\phi_{1}\left(x^{\prime}\right)+\phi_{2}^{2}(x)
$$

where

$$
\phi_{2}=\operatorname{sgn}\left(x_{1}-X\left(x^{\prime}\right)\right)\left(\phi(x)-\phi_{1}\left(x^{\prime}\right)\right)^{\frac{1}{2}} .
$$

Correspondingly,

$$
\chi_{k}^{2} \phi(x)=\chi_{k}^{2} \chi\left(\frac{\left|x^{\prime}\right|}{2 \epsilon}\right) \phi_{1}\left(x^{\prime}\right)+\left(\chi_{k}(x) \phi_{2}(x)\right)^{2} .
$$

Hence we can apply the induction hypothesis to $\chi\left(\frac{\left|x^{\prime}\right|}{2 \epsilon}\right) \phi_{1}\left(x^{\prime}\right)$ and obtain the decomposition of $\chi_{k}^{2} \phi(x)$ into a sum of squares provided we can prove the following

Lemma 5.5. With the above notations we have $\phi_{1} \in C^{3,1}(B(0,2 \epsilon))$ and $\phi_{2} \in C^{2,1}(D)$. More precisely, we obtain bounds which depend only on $\epsilon$, namely

$$
\begin{array}{cc}
\left|\nabla^{j} \phi_{1}\left(x^{\prime}\right)\right| \leq c(\epsilon), \quad j=0,1,2,3,4 \quad & \left|x^{\prime}\right| \leq 2 \epsilon, \\
\left|\nabla^{j} \phi_{2}(x)\right| \leq c(\epsilon), \quad j=0,1,2,3 \quad x \in D .
\end{array}
$$

Proof. To keep the proof simple we give only the qualitative arguments. These can be easily turned into explicit quantitative arguments. Compute first the derivatives of $\phi_{1}$ :

$$
\begin{aligned}
\partial_{i} \phi_{1}\left(x^{\prime}\right) & =\partial_{i} \phi\left(X\left(x^{\prime}\right), x^{\prime}\right)+\partial_{1} \phi\left(X\left(x^{\prime}\right), x^{\prime}\right) \partial_{i} X\left(x^{\prime}\right) \\
& =\left(\partial_{i} \phi\right)\left(X\left(x^{\prime}\right), x^{\prime}\right) .
\end{aligned}
$$

Since both $X$ and $\partial_{i} \phi$ are of class $C^{2,1}$ it follows that $\partial_{i} \phi_{1}$ are of class $C^{2,1}$, i.e. $\phi_{1}$ is of class $C^{3,1}$.

To show that $\phi_{2} \in C^{2,1}$ we can assume without any restriction in generality that $\phi_{1}=0$. We can also make a $C^{2,1}$ change of variable

$$
\left(x_{1}, x^{\prime}\right) \rightarrow\left(x_{1}-X\left(x^{\prime}\right), x^{\prime}\right),
$$

which reduces the problem to the case when $X \equiv 0$ at the expense of lowering the regularity of $\phi$ to $\phi \in C^{2,1}$ and $\partial_{x_{1}} \phi \in C^{2,1}$. Then $\phi\left(0, x^{\prime}\right)=\nabla \phi\left(0, x^{\prime}\right)=0$ therefore by (31) we can represent it in the form

$$
\phi(x)=x_{1}^{2} \psi(x), \quad \psi \geq \frac{1}{4} .
$$

Thus $\phi_{2}=x_{1} \sqrt{\psi}$ therefore it suffices to establish that

$$
\psi \in C^{1,1}, \quad{ }_{18} x_{1} \psi \in C^{2,1} .
$$


More generally, we claim that

$$
\partial_{x_{1}} \phi \in C^{k-1}, \phi\left(0, x^{\prime}\right)=0 \Longrightarrow \frac{\phi(x)}{x_{1}} \in C^{k-1} .
$$

The $x^{\prime}$ derivatives can be factored out, so it remains to look at the one dimensional problem. But this is easily solved using Taylor's formula of order $k$ around $x_{1}=0$.

This concludes the proof of Proposition 1.1.

Proof of Proposition 1.9. We use again induction with respect to the dimension. We begin the induction in dimension 0 , when the result is trivial. It remains to do the induction step. We start with a bound for the gradient of $b$.

Lemma 5.6. Let $b, a_{j}$ be functions satisfying

$$
|b| \leq \max _{j}\left|a_{j}\right|, \quad\left|\nabla^{2} b\right|,\left|\nabla^{2} a_{j}\right| \leq 1 .
$$

Then

$$
|\nabla b| \leq \frac{13}{4} \max _{j}\left|\nabla a_{j}\right|+\left|a_{j}\right|^{\frac{1}{2}}
$$

Proof. Without any restriction in generality we can assume that the dimension is 1 . Let $x \in \mathbb{R}$. Suppose that

$$
\left|a_{j}(x)\right| \leq \delta^{2}, \quad\left|a_{j}^{\prime}(x)\right| \leq \delta .
$$

Due to the bound on the second derivatives, this implies that

$$
\left|a_{j}(y)\right| \leq \frac{5}{2} \delta^{2} \quad \text { for }|y-x| \leq \delta .
$$

Hence we get the same bound for $b$. By the mean value theorem, $\left|b^{\prime}(y)\right|<\frac{5}{4} \delta$ for some $|y-x| \leq \delta$. Using the bound on the second derivative of $b$ we therefore obtain $\left|b^{\prime}(x)\right| \leq \frac{13}{4} \delta$.

For each $x$ we define

$$
r_{j}(x)=\left|\nabla a_{j}(x)\right|+\left|a_{j}\right|^{\frac{1}{2}}, \quad r(x)=\max _{j} r_{j}(x) .
$$

Then it is a simple exercise to show that $r_{j}, r$ are slowly varying. Hence we can apply Lemma 5.4 to get a locally finite covering

$$
\text { supp } a=\bigcup B\left(x_{k}, \epsilon r\left(x_{k}\right)\right),
$$

with a corresponding partition of unit,

$$
1=\sum \chi_{k} \quad \text { in }\{r>0\} .
$$

with $\chi_{k}$ supported in $B\left(x_{k}, \epsilon r\left(x_{k}\right)\right)$. Here $\epsilon$ is a small fixed parameter. We can replace the function $r$ by

$$
\tilde{r}(x)=\sum_{19} r\left(x_{k}\right) \chi_{k} .
$$


In doing this we preserve its size while gaining differentiability

$$
\tilde{r}(x) \approx r(x), \quad\left|\partial^{\alpha} \tilde{r}(x)\right| \lesssim r^{1-|\alpha|} .
$$

We decompose the functions $a_{j}, b$ into

$$
a_{j}=\sum \chi_{k} a_{j}, \quad b=\sum \chi_{k} b .
$$

Then the localized components $\chi_{k} a_{j}, \chi_{k} b$ still have bounded second order derivatives and satisfy the inequality

$$
\left|\chi_{k} b\right| \leq \sum_{j}\left|\chi_{k} a_{j}\right|
$$

Hence, due to the finite intersection property, it suffices to prove the result in a ball $B=B\left(x_{k}, \epsilon r\left(x_{k}\right)\right)$. By rescaling and translation we can assume that $x_{k}=0, r(0)=1$. Then both $a_{j}$ and $b$ are of class $C^{1,1}$ in $B$ with uniform bounds.

According to the definition of $r(x)$ we need to consider two cases:

i) We have $\left|a_{j}(0)\right| \geq \frac{1}{2}$ for some $j$. Hence there exists some $j$ so that $\left|a_{j}\right| \gtrsim \frac{1}{4}$ in $B$. Then within $B$ we represent $b$ as $b=c_{j} a_{j}$. The function $c_{j}$ is of class $C^{1,1}$ in $B$, which concludes the proof.

ii) We have $\left|\nabla a_{j}(0)\right|>\frac{1}{2}$ for some $j$. Then without any restriction in generality we can assume that $\left|\partial_{1} a_{1}(0)\right|>\frac{1}{2}$; If $\epsilon$ is sufficiently small this in turn implies that $\left|\partial_{1} a_{1}(0)\right|>\frac{1}{4}$ in $4 B$. Since $a_{1}$ is $C^{1,1}$ we can make a $C^{1,1}$ change of coordinates in $4 B$ so that in the new coordinates we have $a_{1}=x_{1}$.

Now we use the induction hypothesis on $x_{1}=0$ to get

$$
b\left(0, x^{\prime}\right)=\sum_{j>1} c_{j}\left(x^{\prime}\right) a_{j}\left(0, x^{\prime}\right) .
$$

with $c_{j}\left(x^{\prime}\right)$ satisfying (12). We want to extend the quotients $c_{j}$ to $B$ so that they still satisfy (12). To achieve this consider the slowly varying function $r^{j}$ associated to $a_{j}$ and a corresponding partition of unit $\chi_{k}^{j}$ associated to a locally finite covering with balls $B\left(x_{k}^{j}, \epsilon r^{j}\left(x_{k}^{j}\right)\right)$. Then we define the function

$$
\chi^{j}=\sum_{B\left(x_{k}^{j}, \epsilon r^{j}\left(x_{k}^{j}\right)\right) \cap\left\{x_{1}=0\right\} \neq \emptyset} \chi_{k}^{j}
$$

which satisfies the bounds

$$
\left|\partial^{\alpha} \chi_{j}(x)\right| \leq c_{\alpha}\left(r^{j}(x)\right)^{-|\alpha|} .
$$

In addition, observe that within the support of $\chi^{j}$ we must have $r^{j}(x) \approx r^{j}\left(0, x^{\prime}\right)$.

Hence, if we set

$$
c_{j}(x)=c_{j}\left(x^{\prime}\right) \chi^{j}(x)
$$

then the function $c_{j}$ not only satisfies (12), but also has some better differentiability properties as a function of $x_{1}$. More precisely, one can verify that

$$
\left|\nabla\left(c_{j} a_{j}\right)\right|,\left|\partial_{1} \nabla\left(c_{j} a_{j}\right)\right| \lesssim 1
$$


Observe, however, that we cannot bound second derivatives of the product with respect to $x^{\prime}$. This allows us to define

$$
c_{1}(x)=\frac{b(x)-\sum_{j>1} c_{j} a_{j}}{x_{1}} .
$$

The numerator vanishes when $x_{1}=0$ and satisfies bounds similar to (34), therefore after the division we get $c_{1}$ Lipschitz. This concludes the proof.

Proof of Proposition 1.12. This is an inductive argument with respect to the dimension which follows the same procedure as in the proof of Proposition 1.9. The replacement of Lemma 5.6 is now

Lemma 5.7. Let $b, a_{j}$ be functions satisfying

$$
b \leq \max _{j}\left|a_{j}\right|, \quad\left|\nabla^{2} b\right|,\left|\nabla^{2} a_{j}\right| \leq 1 .
$$

Then

$$
|\nabla b| \leq 9\left(|b|^{\frac{1}{2}}+\max _{j}\left|\nabla a_{j}\right|+\left|a_{j}\right|^{\frac{1}{2}}\right) .
$$

The proof is almost identical. For each $x$ we define

$$
r(x)=|b(x)|^{\frac{1}{2}}+\max _{j}\left|\nabla a_{j}(x)\right|+\left|a_{j}\right|^{\frac{1}{2}} .
$$

Then $r$ is slowly varying. Arguing as before, we can use the same reduction argument involving the partition of unit in Lemma 5.4. Thus after rescaling and translation it suffices to obtain the desired decomposition for $b$ in a ball $B=B(0, \epsilon)$, under the assumption that $r(0)=1$. This time we need to consider three cases:

i) $|a(0)|,|\nabla a(0)| \ll 1$. Then $b(0) \approx-1$. Hence $b$ is negative in $B$, therefore our decomposition in $B$ is $b_{0}=b, b_{1}=0$.

The remaining two cases correspond to the two cases in the proof of Proposition 1.9.

ii) $|a(0)|=O(1)$. Then $|b| \leq|a|$ in $B$, so the desired decomposition is $b_{0}=0$, $b_{1}=b$.

iii) $|a(0)| \ll|\nabla a(0)|=O(1)$. Arguing as in the proof of Proposition 1.9, the problem reduces to the case when

$$
a_{1}=x_{1}, \quad\left|a_{j}\right|,\left|\nabla a_{j}\right|, b,|\nabla b| \lesssim 1 .
$$

Using the induction hypothesis we decompose $b$ on $x_{1}=0$,

$$
b\left(0, x^{\prime}\right)=b_{0}\left(0, x^{\prime}\right)+b_{1}\left(0, x^{\prime}\right) .
$$

Then we extend this decomposition by setting

$$
b_{0}\left(x_{1}, x^{\prime}\right)=b_{0}\left(0, x^{\prime}\right),
$$

which is still negative. It remains to verify that

$$
\left|b_{1}\left(x_{1}, x^{\prime}\right)\right| \leq \sum_{21}\left|a_{j}\left(x_{1}, x^{\prime}\right)\right| \quad \text { in } B .
$$


Indeed,

$$
\begin{aligned}
\left|b_{1}\left(x_{1}, x^{\prime}\right)\right| & \leq\left|b_{0}\left(0, x^{\prime}\right)\right|+\left|b\left(x_{1}, x^{\prime}\right)-b\left(0, x^{\prime}\right)\right| \leq \sum\left|a_{j}\left(0, x^{\prime}\right)\right|+c\left|x_{1}\right| \\
& \leq \sum\left|a_{j}\left(x, x^{\prime}\right)\right|+c\left|x_{1}\right| .
\end{aligned}
$$

\section{AN APPLICATION TO LOCAL SOLVABILITY}

The aim of this section is to consider a classical result on local solvability for partial differential operators with smooth coefficients, and extend it to the case when the coefficients have limited smoothness. Consider a partial differential operator $P(x, D)$ of order $m$ in $\Omega \subset \mathbb{R}^{n}$. Then we say that $P$ is locally solvable with loss of $k$ derivatives if for any $x_{0} \in \Omega$ there is a neighborhood $V$ of $x_{0}$ so that for all $f$ in a finite codimensional subspace of $L^{2}$, supported in $V$ there is a function $u \in H_{l o c}^{m-k}$ solving $P(x, D) u=f$ in $V$. Normally the local solvability is stated with smooth $f$ and $u$, but this does not make sense for nonsmooth coefficients.

The local solvability result we consider refers to principally normal operators of principal type. In the following definitions $p(x, \xi)$ stands for the principal symbol of $P(x, D)$.

Definition 6.1. The operator $P(x, D)$ is of principal type if

$$
\nabla_{\xi} p(x, \xi) \neq 0 \quad \text { on }\{p(x, \xi)=0\} .
$$

Definition 6.2. We say that the operator $P$ is principally normal if

$$
|\{\bar{p}, p\}| \leq c|\xi|^{m-1}|p| .
$$

Here $\{\cdot, \cdot\}$ represents the Poisson bracket of two symbols.

Hörmander [5][7] proved that if $P$ is a principally normal operators of principal type with smooth coefficients then it is locally solvable with loss of one derivative. We obtain here a similar result for operators with $C^{2,1}$ coefficients.

Theorem 6. Suppose $P(x, D)$ is a principally normal operator of principal type with $C^{2,1}$ coefficients. Then it is locally solvable with loss of one derivative.

Remark 6.3. What is needed in the proof, in effect, is that both $p$ and $\{\bar{p}, p\}$ have $C^{1,1}$ coefficients.

Proof. We take $x_{0}=0$ and $V=B(0, \epsilon)$ with $\epsilon$ small. Rescaling by a factor of $\epsilon^{-1}$, we need to prove local solvability for the rescaled operator $P_{\epsilon}(x, D)=\epsilon^{m} P\left(\epsilon x, \epsilon^{-1} D\right)$ in the unit ball. By standard functional analysis arguments the local solvability with loss of one derivative for $P$ follows from a bound for the adjoint operator:

$$
\|v\|_{L^{2}} \lesssim\left\|P_{\epsilon}^{*} v\right\|_{H^{1-m}}+\|v\|_{H^{-1}} \text {. }
$$

for all $v$ supported in $B$. We claim that this estimate holds uniformly in $\epsilon$ for small $\epsilon$. The conjugated operator $P_{\epsilon}^{*}$ is given by

$$
\begin{gathered}
P_{\epsilon}^{*}=\epsilon^{m} \bar{P}\left(\epsilon^{-1} D, \epsilon x\right) . \\
22
\end{gathered}
$$


The lower order terms in $P$ come with factors of $\epsilon$ so they are negligible in (36). Hence without any restriction in generality we can assume that $P$ is homogeneous of order $m$, in which case

$$
P_{\epsilon}^{*}=\bar{P}(D, \epsilon x) .
$$

Replacing $P_{\epsilon}^{*}$ by $Q(D, \epsilon x)$ with

$$
q(x, \xi)=|\xi|^{1-m} \bar{p}(x, \xi) .
$$

the estimate (36) becomes

$$
\|v\|_{L^{2}} \lesssim\|Q(D, \epsilon x) v\|_{L^{2}}+\|v\|_{H^{-1}}
$$

while $q$ is also principally normal,

$$
|\{q, \bar{q}\}| \lesssim|q| .
$$

We write $q=a+i b$. Then (37) follows from the next two estimates. The first one is a Fefferman-Phong type estimate which is a consequence of the principal normality condition.

$$
\|A(D, \epsilon x) v\|_{L^{2}}+\|B(D, \epsilon x) v\|_{L^{2}} \lesssim\|Q(D, \epsilon x) v\|_{L^{2}}+\epsilon\|v\|_{L^{2}} .
$$

The second one is an energy estimate which follows from the principal type condition.

$$
\|v\|_{L^{2}} \lesssim\|A(D, \epsilon x) v\|_{L^{2}}+\|B(D, \epsilon x) v\|_{L^{2}}+\|v\|_{H^{-1}} .
$$

The proof of (40) relies on a standard argument which requires only one derivative on the coefficients, see [11]. The idea is as follows. Given some $\xi_{0} \in \mathbb{R}^{n}$ we can find some direction, say $\xi_{1}$, so that either $\partial_{\xi_{1}} a\left(0, \xi_{0}\right) \neq 0$ or $\partial_{\xi_{1}} b\left(0, \xi_{0}\right) \neq 0$. If $\epsilon$ is small then the same happens for $x$ in $B(0, \epsilon)$. Hence either $A$ or $B$ is microhyperbolic at $\xi_{0}$ in the direction $x_{1}$ and the estimate (40) follows microlocally near $\xi_{0}$. The microlocal estimates can be put together using a conical partition of unit in frequency.

It remains to prove (39). This is our main point here. The estimate (39) can be rescaled back to

$$
\|A(D, x) v\|_{L^{2}}+\|B(D, x) v\|_{L^{2}} \lesssim\|Q(D, x) v\|_{L^{2}}+\|v\|_{L^{2}}
$$

The support information for $v$ is not useful here. To prove this compute

$$
\begin{aligned}
\|Q(D, x) v\|_{L^{2}} & =\|A(D, x) v\|_{L^{2}}+\|B(D, x) v\|_{L^{2}}+2 \Re<A(D, x) v, i B(D, x) v> \\
& =\|A(D, x) v\|_{L^{2}}+\|B(D, x) v\|_{L^{2}}+<C v, v>
\end{aligned}
$$

where

$$
C=i(A(x, D) B(D, x)-B(x, D) A(D, x)) .
$$

Then the pdo calculus for operators with rough symbols (see [11]) shows that, modulo an $L^{2}$ bounded error term, we have $C=C(D, x)$ where

$$
c(x, \xi)=a_{x \xi} b-b_{x \xi} a+\{a, b\} .
$$

This computation requires only $C^{1,1} S^{1}$ regularity for the symbols $a$ and $b$. However, the additional derivative on the coefficients implies that also $c \in C^{1,1} S^{1}$. To conclude it suffices to verify that

$$
\|C(D, x) v\|_{L^{2}} \lesssim\|A(D, x) v\|_{L^{2}}+\|B(D, x) v\|_{L^{2}}+\|v\|_{L^{2}} .
$$


The principal normality condition on $q=a+i b$ gives

$$
|c| \lesssim|a|+|b| \text {. }
$$

Also the operator $C(D, x)-C(x, D)$ is $L^{2}$ bounded, and similarly for $A$ and $B$. Then (41) follows from Corollary 1.11.

\section{An APPLiCATion to UNiQUe CONTINUATION}

Consider a partial differential operator $P(x, D)$ of order $m$ and an oriented surface $S=\{\phi=0\}$ in $\mathbb{R}^{n}$. We say that the unique continuation property across $S$ holds for solutions $u$ to $P(x, D) u=0$ if for each $x_{0} \in S$ and $u$ a solution to $P(x, D) u=0$ near $x_{0}, u=0$ in $\{\phi>0\}$ near $x_{0}$ implies that $u=0$ near $x_{0}$. In other words, if $u$ vanishes on one side of $S$ then it must also vanish on the other side. One can also view the unique continuation property as an uniqueness result for the Cauchy problem corresponding to the equation $P(x, D) u=0$ with Cauchy data on $S$. This is formally equivalent to an existence result for the Cauchy problem corresponding to the adjoint operator $P^{*}$. Note that the orientation of the surface is important, if one has unique continuation in one direction this says nothing about unique continuation in the opposite direction.

The interesting question in unique continuation is, given an operator $P$, to determine the surfaces $S$ across which unique continuation holds for solutions $u$ to $P(x, D) u=0$. This problem is reasonably well understood, so we proceed to state one of the main results. Some definitions are necessary. We denote by $p(x, \xi)$ the principal symbol of $P(x, D)$. If $\phi$ is the defining function for the surface $S$ we introduce the symbol

$$
p_{\phi}=p(x, \xi+i \tau \nabla \phi)
$$

Definition 7.1. We say that the oriented surface $S$ is strongly pseudoconvex with respect to the operator $P$ if

(i) $\{\bar{p},\{p, \phi\}\}>0$ on $p=\{p, \phi\}=0$

(ii) $\left\{\overline{p_{\phi}}, p_{\phi}\right\}>0$ on $p_{\phi}=\left\{p_{\phi}, \phi\right\}=0, \tau>0$.

Then (see [7] and references therein):

Theorem 7. (Hörmander) Let $P$ be a principally normal operator with smooth coefficients. If $S$ is strongly pseudoconvex with respect to $P$ then unique continuation across $S$ holds for solutions $u$ to $P(x, D) u=0$.

The question we want to address is to what extent the regularity of the coefficients can be relaxed in this result. Two cases when the principal normality condition for $P$ is trivially satisfied are (i) when $P$ is elliptic and (ii) when the principal symbol of $P$ is real. In both cases, the unique continuation result is known to hold for operators with merely $C^{1}$ coefficients. Here we consider the general case. Our result is

Theorem 8. Let $P$ be a principally normal operator with $C^{2,1}$ coefficients. If $S$ is strongly pseudoconvex with respect to $P$ then unique continuation across $S$ holds for solutions $u$ to $P(x, D) u=0$. 
Remark 7.2. Again, what is needed in the proof is that both $p$ and $\left\{\overline{p_{\phi}}, p_{\phi}\right\}$ have $C^{1,1}$ coefficients.

The unique continuation result is a consequence of certain Carleman estimates. These are a one parameter family of weighted estimates, of the form

$$
\tau\left\|e^{\tau \psi} u\right\|_{H_{\tau}^{m-1}} \leq C \tau\left\|e^{\tau \psi} P(x, D) u\right\|_{L^{2}} \quad \tau>\tau_{0}>0 .
$$

where the norms of the weighted spaces $H_{\tau}^{m}$ are defined as

$$
\|u\|_{H_{\tau}^{m}}^{2}=\|u\|_{H^{m}}^{2}+\tau^{2 m}\|u\|_{L^{2}}^{2}
$$

which corresponds to the usual norms if we interpret $\tau$ as a derivative. The essential feature of these estimates is that the constant $C>0$ does not depend on $\tau$.

The function $\psi$ is chosen to have level sets close to the level sets of $\phi$, but more convexity across level sets. In practice, a good choice is to take $\psi$ a small $C^{2}$ perturbation of $e^{\mu \phi}$, with $\mu$ large enough. Then (see [7]) $\psi$ will satisfy the strong pseudoconvexity condition for functions:

Definition 7.3. We say that the function $\psi$ is strongly pseudoconvex with respect to the operator $P$ if

$$
\frac{1}{i}\left\{p_{\psi}, \overline{p_{\psi}}\right\} \geq C^{-1} \tau(\tau+|\xi|)^{2(m-1)}-C(\tau+|\xi|)^{m-1}\left|p_{\psi}\right| .
$$

The fact that Carleman estimates imply unique continuation is well explained in the literature. Instead, let us focus on the proof of the estimate (42). With the substitution $v=e^{\tau \psi} u$ (42) becomes

$$
\tau\|v\|_{H_{\tau}^{m-1}} \leq C \tau\left\|P_{\psi} v\right\|_{L^{2}} \quad \tau>\tau_{0}>0
$$

where the conjugated operator $P_{\psi}$ is given by

$$
P_{\psi}=e^{\tau \psi} P(x, D) e^{-\tau \psi}=P(x, D+i \tau \nabla \psi) .
$$

and has principal symbol $p_{\psi}$. The lower order terms are negligible in (44) if $\tau$ is sufficiently large. Set

$$
q(x, \xi, \tau)=p_{\psi}(x, \xi, \tau)\left(\xi^{2}+\tau^{2}\right)^{-\frac{m-1}{2}} .
$$

Then $q$ is a pseudodifferential operator of order 1 , and we need to show that

$$
\tau\|v\|_{L^{2}} \leq C\|Q(x, D, \tau) v\|_{L^{2}} . \quad \tau>\tau_{0}>0
$$

On the other hand $Q \in C^{2,1} S^{1}$ and from (43) we get

$$
\frac{1}{i}\{q, \bar{q}\} \geq C^{-1} \tau+C|q| \text {. }
$$

We decompose $q$ into its real and imaginary part,

$$
q=a+i b .
$$

Then, computing as in the previous section,

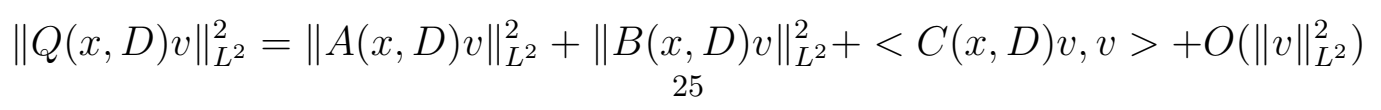


where

$$
c(x, \xi)=a_{x \xi} b-b_{x \xi} a+\{a, b\} .
$$

Hence it remains to prove that

$$
C^{-1} \tau\|v\|_{L^{2}} \leq\|A(x, D) v\|_{L^{2}}^{2}+\|B(x, D) v\|_{L^{2}}^{2}+<C(x, D) v, v>.
$$

But the pseudoconvexity condition (46) implies that

$$
c \geq C^{-1}|\tau|-C(|a|+|b|)
$$

therefore this follows from Corollary 1.11.

\section{REFERENCES}

[1] Jean-Michel Bony. Sur l'inégalité de Fefferman-Phong. In Seminaire: Équations aux Dérivées Partielles, 1998-1999, pages Exp. No. III, 16. École Polytech., Palaiseau, 1999.

[2] Jean-Marc Delort. F.B.I. transformation. Springer-Verlag, Berlin, 1992. Second microlocalization and semilinear caustics.

[3] C. Fefferman and D. H. Phong. On positivity of pseudo-differential operators. Proc. Nat. Acad. Sci. U.S.A., 75(10):4673-4674, 1978.

[4] Charles L. Fefferman. The uncertainty principle. Bull. Amer. Math. Soc. (N.S.), 9(2):129-206, 1983.

[5] Lars Hörmander. Differential operators of principal type. Math. Ann., 140:124-146, 1960.

[6] Lars Hörmander. Pseudo-differential operators and non-elliptic boundary problems. Ann. of Math. (2), 83:129-209, 1966.

[7] Lars Hörmander. The analysis of linear partial differential operators. I-IV. Springer-Verlag, Berlin, 1985. Fourier integral operators.

[8] Lars Hörmander. The analysis of linear partial differential operators. I-IV. Springer-Verlag, Berlin, second edition, 1990. Distribution theory and Fourier analysis.

[9] P. D. Lax and L. Nirenberg. On stability for difference schemes: A sharp form of Gärding's inequality. Comm. Pure Appl. Math., 19:473-492, 1966.

[10] Daniel Tataru. Strichartz estimates for operators with nonsmooth coefficients and the nonlinear wave equation. Amer. J. Math., 122(2):349-376, 2000.

[11] Michael E. Taylor. Pseudodifferential operators and nonlinear PDE. Birkhäuser Boston Inc., Boston, MA, 1991.

Department of Mathematics, Northwestern University 